\title{
Article \\ Generation of a Novel Transgenic Zebrafish for Studying Adipocyte Development and Metabolic Control
}

\author{
Yousheng Mao ${ }^{1}{ }^{\mathbb{D}}$, Kwang-Heum Hong ${ }^{1}$, Weifang Liao ${ }^{1} \mathbb{D}$, Li Li ${ }^{1}$, Seong-Jin Kim ${ }^{2}$, Yinyi Xiong ${ }^{1}$, In-Koo Nam ${ }^{3,4}$, \\ Seong-Kyu Choe ${ }^{1,2,4,5, * \mathbb{D}}$ and Seong-Ae Kwak ${ }^{6, *}$
}

1 Department of Medicine, Graduate School, Wonkwang University, Iksan 54538, Korea; maoyousheng1992@163.com (Y.M.); asdfdfghgh@naver.com (K.H.); 15070285697@163.com (W.L.); lilidaibaoli@163.com (L.L.); x1992889@gmail.com (Y.X.)

2 Department of Biomedical Science, Graduate School, Wonkwang University, Iksan 54538, Korea; mbunker1.21@gmail.com

3 Institute of Brain Science, Wonkwang University, Iksan 54538, Korea; 8012645@hanmail.net

4 Department of Microbiology, School of Medicine, Wonkwang University, Iksan 54538, Korea

5 Institute of Wonkwang Medical Science, Wonkwang University, Iksan 54538, Korea

6 Hanbang Cardio-Renal Research Center, Wonkwang University, Iksan 54538, Korea

* Correspondence: seongkyu642@wku.ac.kr (S.-K.C.); seongae642@wku.ac.kr (S.K.); Tel.: +82-63-850-6975 (S.-K.C.); +82-63-850-6447 (S.K.); Fax: +82-63-851-5680 (S.-K.C.)

Citation: Mao, Y.; Hong, K.; Liao, W.; Li, L.; Kim, S.; Xiong, Y.; Nam, I.-K.; Choe, S.-K.; Kwak, S. Generation of a Novel Transgenic Zebrafish for Studying Adipocyte Development and Metabolic Control. Int. J. Mol. Sci. 2021, 22, 3994. https://doi.org/ $10.3390 /$ ijms22083994

Academic Editor: Antonino Germana

Received: 23 March 2021

Accepted: 10 April 2021

Published: 13 April 2021

Publisher's Note: MDPI stays neutral with regard to jurisdictional claims in published maps and institutional affiliations.

Copyright: (c) 2021 by the authors. Licensee MDPI, Basel, Switzerland. This article is an open access article distributed under the terms and conditions of the Creative Commons Attribution (CC BY) license (https:// creativecommons.org/licenses/by/ $4.0 /)$.

\begin{abstract}
Zebrafish have become a popular animal model for studying various biological processes and human diseases. The metabolic pathways and players conserved among zebrafish and mammals facilitate the use of zebrafish to understand the pathological mechanisms underlying various metabolic disorders in humans. Adipocytes play an important role in metabolic homeostasis, and zebrafish adipocytes have been characterized. However, a versatile and reliable zebrafish model for long-term monitoring of adipose tissues has not been reported. In this study, we generated stable transgenic zebrafish expressing enhanced green fluorescent protein (EGFP) in adipocytes. The transgenic zebrafish harbored adipose tissues that could be detected using GFP fluorescence and the morphology of single adipocyte could be investigated in vivo. In addition, we demonstrated the applicability of this model to the long-term in vivo imaging of adipose tissue development and regulation based on nutrition. The transgenic zebrafish established in this study may serve as an excellent tool to advance the characterization of white adipose tissue in zebrafish, thereby aiding the development of therapeutic interventions to treat metabolic diseases in humans.
\end{abstract}

Keywords: transgenic zebrafish; adipose tissue; live imaging; obesity

\section{Introduction}

The incidence of obesity and overweight individuals has been increasing worldwide over the past decades, and these two issues have attained a pandemic status [1]. Obesity can lead to the development of numerous health problems, including insulin resistance (IR), type 2 diabetes mellitus (T2DM), non-alcoholic fatty liver disease (NAFLD), cardiovascular disorders, and hypertension [2-4], and has, therefore, attracted considerable attention. In particular, visceral fat accumulation is considered as a major risk for the development of IR which then may facilitate the development of obesity-related metabolic syndrome [4,5]. In addition, reactive oxygen species seems to be a key player in the development of IR and the subsequent development of NAFLD or T2DM $[4,6,7]$. However, due to the complexity involving genetic, environmental, and behavioral effects to the development of obesity, the precise pathogenetic mechanisms leading to NAFLD and T2DM are not fully understood. Clinical evidence showed that some subjects with high degree of obesity can be considered metabolically healthy, suggesting that obesity per se may not always cause metabolic syndrome [8]. Moreover, limited adipose tissue expansion may also play a critical role in the development of T2DM and NAFLD [4,9]. Based on the adipose tissue expandability 
hypothesis, ectopic lipid load in non-adipocyte cells may induce persistent lipotoxicity such as IR, apoptosis, and inflammation which then forms the basis for the onset of T2DM and NAFLD. Therefore, integration of complex interactions between adipose tissues and other metabolic organs is pivotal to the understanding of energy balance and the development of obesity [10].

Since no animal model completely reflects biological pathways and diseases in humans, different model systems are utilized to compensate for intrinsic differences among them. Most of the current information regarding adipose tissue has been obtained using mammalian in vivo and in vitro models [9]. Zebrafish have attracted the attention of researchers because they provide an evolutionarily conserved and optically transparent system for developmental research [11]. In addition, zebrafish exhibit several advantages for metabolic research, including largely conserved physiology and anatomy compared with mammals, a broad range of genetic manipulations, and large-scale phenotypic screens [12]. The adipose tissues of zebrafish and mammals exhibit great similarities; first, human and zebrafish adipocytes contain large lipid droplets [13-15]. Second, they exhibit an energy storage function $[15,16]$. Third, human and zebrafish adipocytes exhibit a similar pattern of gene expression in terms of adipocyte differentiation and endocrine regulation $[15,17,18]$. Therefore, zebrafish have the potential to be used as an in vivo model to obtain new insights into the intrinsic mechanism of white adipose tissues, which may provide a new information of obesity and related comorbidity.

Based on the anatomical position, zebrafish adipose tissue can be divided into two major types; internal adipose tissue (IAT) and subcutaneous adipose tissue (SAT) [19]. IAT includes visceral and non-visceral adipose tissues such as paraosseal adipose tissue. SAT includes cranial (for example, ocular adipose tissue), truncal (for example, abdominal, dorsal, and ventral SAT) and appendicular SAT (for example, pectoral and caudal fin ray SAT). At present, in vivo observation of various zebrafish adipose tissues mainly involves the use of lipophilic dyes [20]. However, lipophilic dyes detect lipid droplets and do not necessarily reflect the size or morphology of the whole adipocyte. The accumulation of neutral lipids in some non-adipose tissues may interfere with data interpretation. Furthermore, it is not convenient or suitable for long-term imaging of adipose tissue using in vivo staining. Therefore, the development of a versatile and reliable zebrafish model for long-term monitoring of adipose tissues and adipocytes in vivo would greatly facilitate the examination of adipocyte dynamism to understand pathological progression of metabolic diseases in humans.

Phylogenetic analyses have revealed that fatty acid binding protein 11a (Fabp11a) and Fabp $11 \mathrm{~b}$ are homologous to mammalian fatty acid binding proteins, including FABP4, FABP5, FABP8, and FABP9 [21,22]. In situ hybridization validated that fabp11a, rather than fabp11b, continues to label adipocytes in major adipose depots [15]. These results suggest the selectivity of the fabp11a promoter to potentially be used to label adipocytes in vivo. In this study, we subcloned several different lengths of the fabp 11a promoter region and performed adipocyte-specific transgenesis in zebrafish. We recovered a transgenic zebrafish containing the 1.5 kilobase-long proximal fabp 11a promoter, which was sufficient to drive the expression of EGFP transgene, specifically in adipocytes throughout the adipose depots during development and in adult zebrafish. The transgenic zebrafish exhibited a reliable change in their adipogenic capacity depending on the nutritional status, suggesting a potential utility in live tracking of adipocytes under normal and pathogenic conditions.

\section{Results}

2.1. Proximal fabp11a Promoter Drives Adipocyte-Specific Expression of EGFP Transgene in Transgenic Animal

Our approach to transgenesis using different lengths of the proximal fabp11a promoter generated F1 zebrafish that specifically expressed EGFP in adipocytes (Figure 1a). To further validate whether the EGFP signal in the stable transgenic line, we raised the next generation and examined the EGFP signal in various adipose tissues. To meet our needs, we found that the transgenic zebrafish line expressed EGFP in IAT, including visceral and 
non-visceral adipose tissues (Figure $1 \mathrm{~b}, \mathrm{c}$ ). In addition, the transgenic zebrafish could also mark SAT, including cranial (such as ocular adipose tissue), truncal (such as abdominal, dorsal, and ventral SAT) and appendicular SAT (such as pectoral fin SAT and caudal fin ray SAT) (Figure $1 \mathrm{~b}, \mathrm{c})$. The newly generated transgenic zebrafish line, $\mathrm{Tg}$ (fabp11a: EGFP), could hence reliably display various adipose tissues in vivo.

(a)

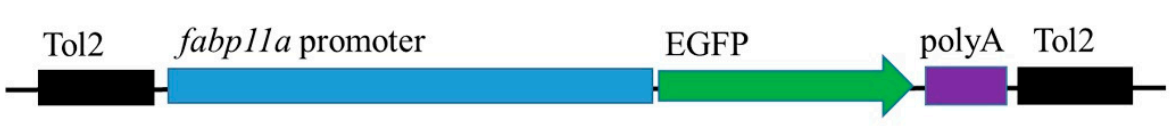

(b)

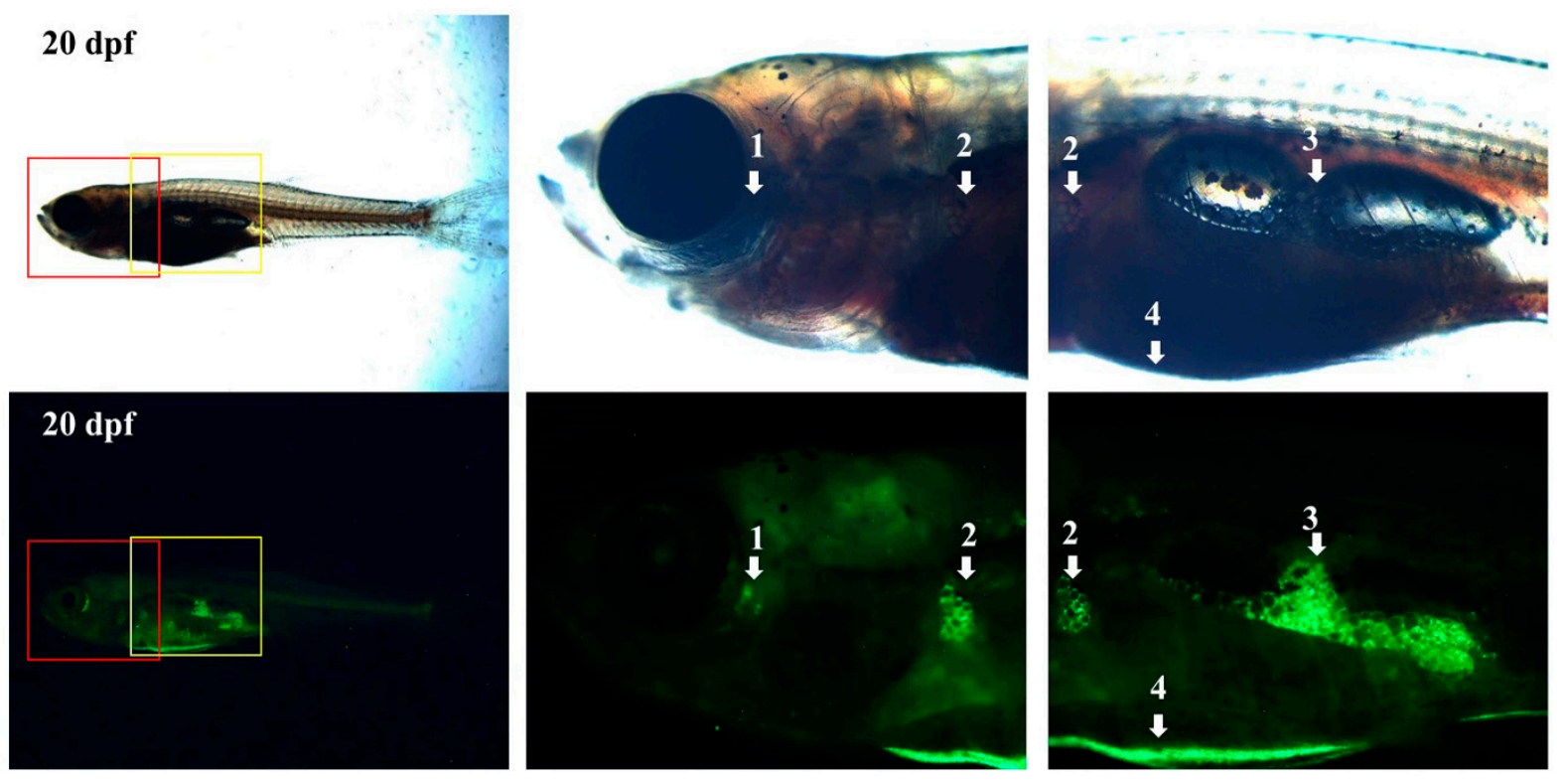

(c)

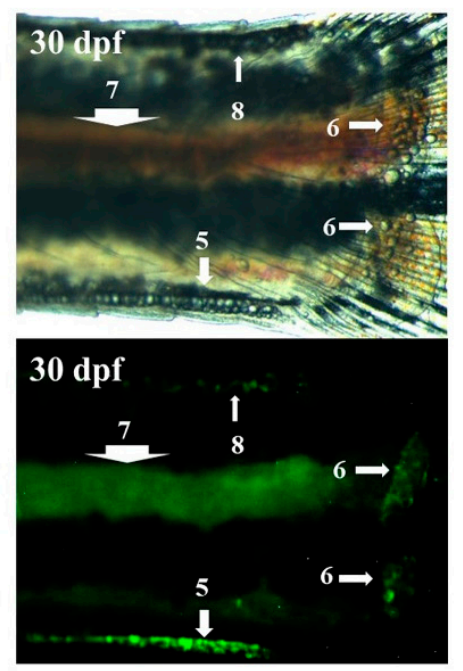

Figure 1. Visualization of adipose tissues in transgenic zebrafish. (a) Schematic drawing of the DNA construct used to generate the zebrafish transgenic line, $\operatorname{Tg}$ (fabp11a: EGFP). (b) Overview of a Tg (fabp11a: EGFP) at $20 \mathrm{dpf}$ and zoom-in views of the boxed areas. Numbers denote adipose depots; 1, OCUAT (ocular adipose tissue); 2, PECSAT (pectoral fin subcutaneous adipose tissue); 3, VAT (visceral adipose tissue); and 4, ASAT (abdominal subcutaneous adipose tissue). (c) Imaging of a $\mathrm{Tg}$ (fabp11a: EGFP) zebrafish tail at $30 \mathrm{dpf}$. 5, VSAT (ventral subcutaneous adipose tissue); 6, CFRSAT (caudal fin ray subcutaneous adipose tissue); 7, POSAT (paraosseal adipose tissue); and 8, DSAT (dorsal subcutaneous adipose tissue). 


\subsection{In Vivo Visualization of Adipocyte Morphology in Transgenic Zebrafish Larvae}

We examined the adipocyte morphology in vivo using the transgenic zebrafish line, Tg (fabp11a: EGFP) (Figure 2a). A typical adipocyte morphology is a single, large lipid droplet with a small nucleus; the adipocytes detected through the expression of EGFP in our transgenic zebrafish contained a large, black center with surrounding area in green (Figure 2a). We presumed that the black center was the lipid droplet, and the green periphery was the cytosol containing EGFP. This was confirmed by Nile Red staining. Nile Red marked the lipid droplets in the adipocytes of wild type (WT) and transgenic zebrafish, whereas the expression of EGFP was broadly detected in the cytosol of the adipocytes of the transgenic zebrafish, differentiating the transgenic zebrafish from their WT siblings (Figure $2 b$ ). Moreover, we were able to detect the morphology of a single adipocyte in vivo using the transgenic zebrafish line (Figure $2 b$ ).

(a)

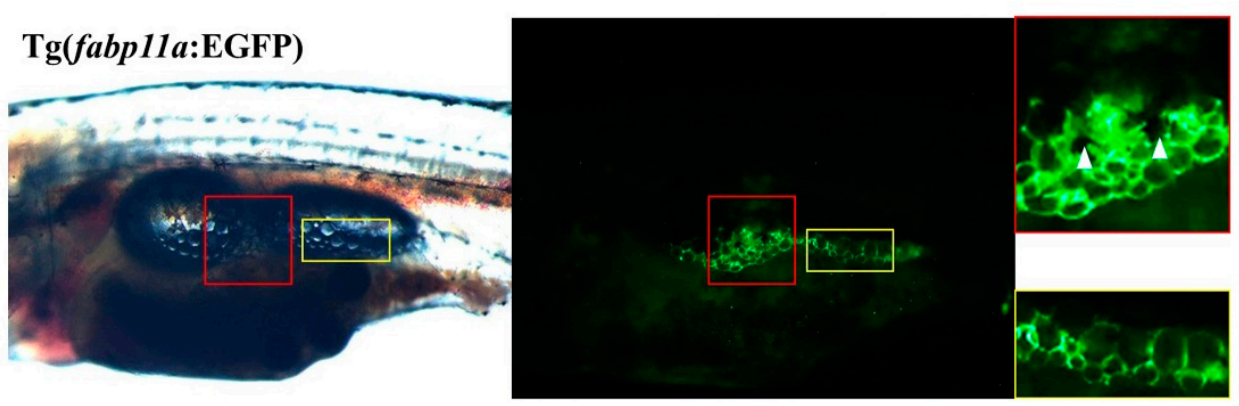

(b)
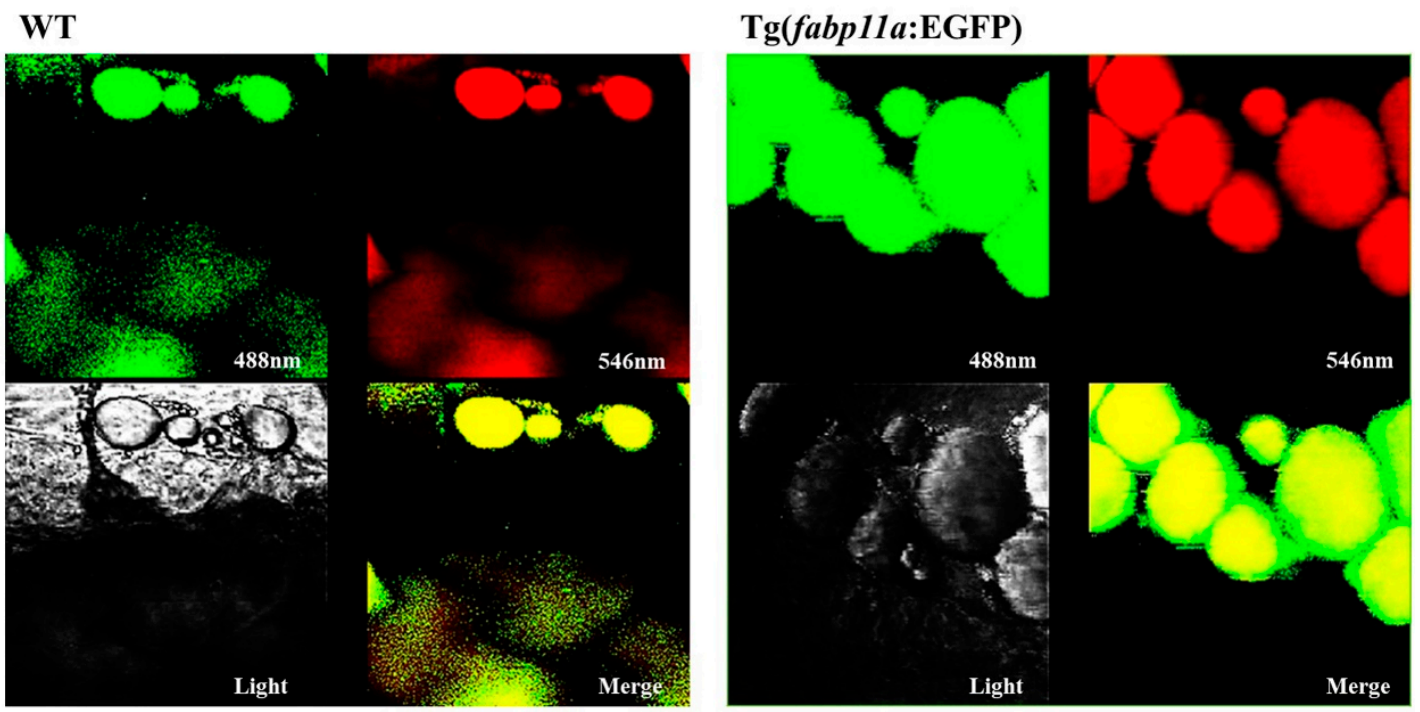

Figure 2. Visualization of adipocyte morphology in the transgenic zebrafish. (a) Images of trunk regions of zebrafish at $18 \mathrm{dpf}$ and zoom-in views of the boxed areas. Red colored boxes show an area of pancreatic adipose tissue with multilayered adipocytes. Yellow colored boxes show an area of adipose tissue with single layer of adipocytes. White arrowheads indicate pigment cells. (b) Confocal images of adipocytes after Nile Red staining which is shown in both green and red. Green fluorescent signals are from both EGFP in the cytoplasm and Nile Red-stained lipid droplet, whereas red fluorescent signal is only from lipid droplet. Merged images show yellow lipid droplet and green cytoplasm.

2.3. In Vivo Monitoring of Transgenic Zebrafish Larvae Revealed Asymmetrical Development of Visceral Adipose Tissues

As our newly generated transgenic zebrafish could be used to detect adipose tissues as well as a single adipocyte, we decided to monitor adipose tissue development in vivo. After monitoring at least $50 \mathrm{Tg}$ (fabp11a: EGFP) larvae continuously from 5 to $30 \mathrm{dpf}$, we confirmed a previously reported finding on the development of adipose tissues in zebrafish; the earliest emerging adipocyte is a visceral adipocyte that appears adjacent to 
the pancreas in a relatively constant position at approximately 9-10 dpf (Figure 3a). In addition, we found that the development and distribution of visceral adipose tissues in zebrafish are asymmetrical; right lateral visceral adipose tissue emerges at least 2 days earlier (Figure 3a,b) and grows much faster than left lateral one (Figure 3b,c). These results clearly suggested that the newly generated transgenic zebrafish line may be useful for continuously monitoring adipose tissue development and may potentially enable to investigate adipocyte hypertrophy and hyperplasia in vivo.

(a)
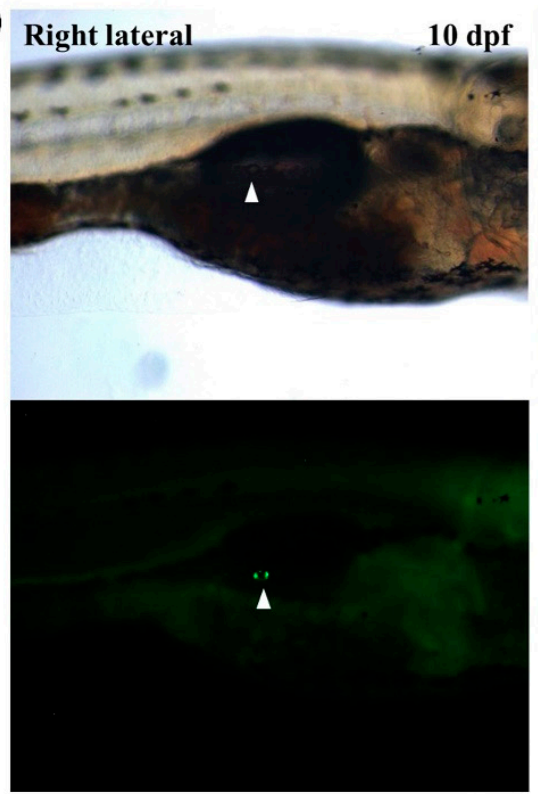

(b)

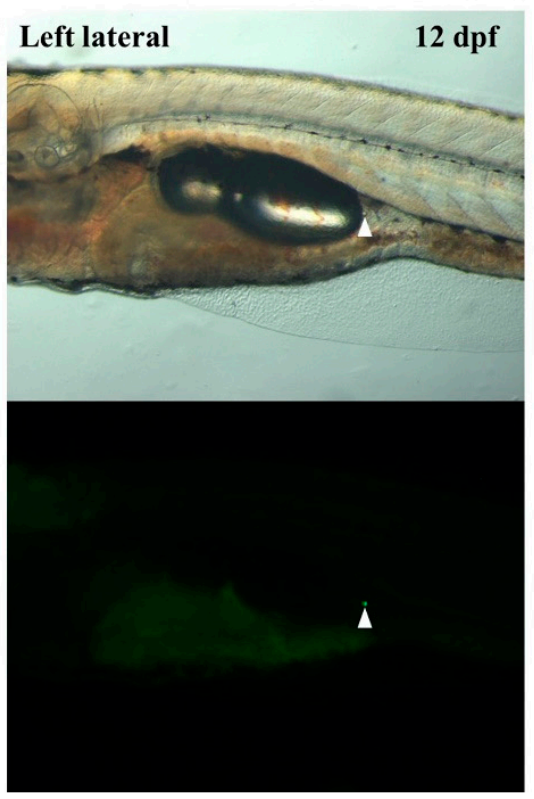

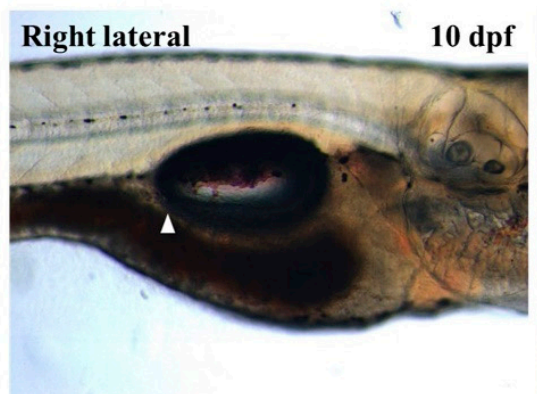
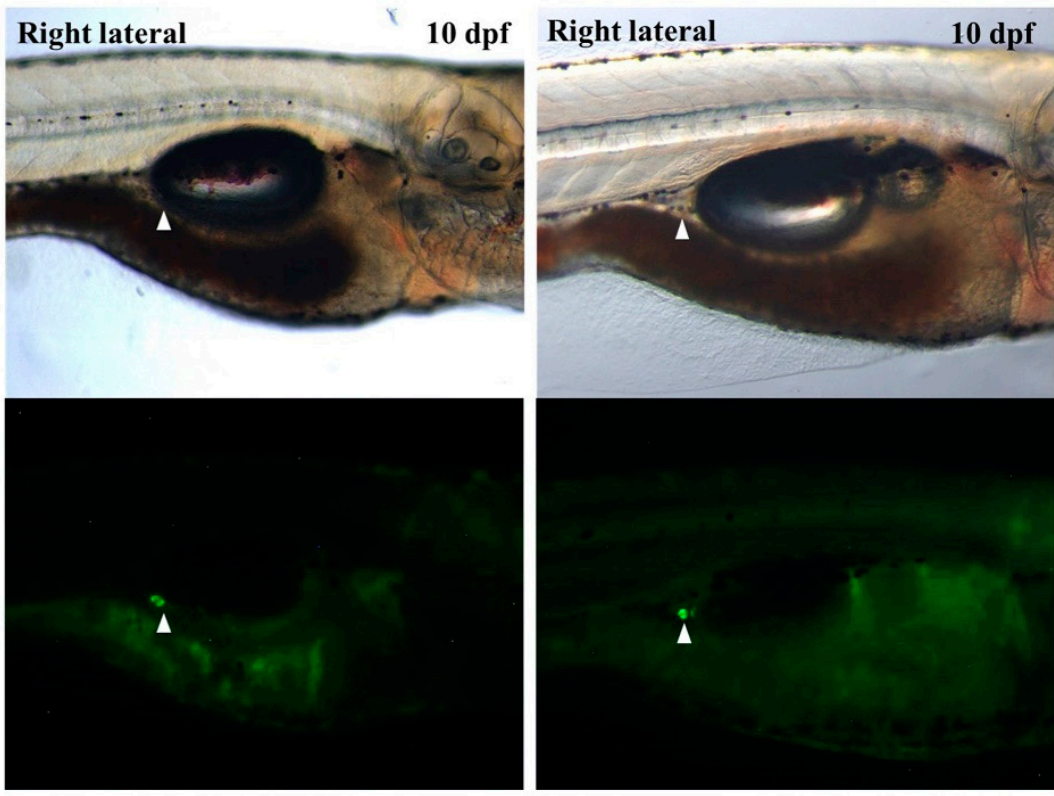

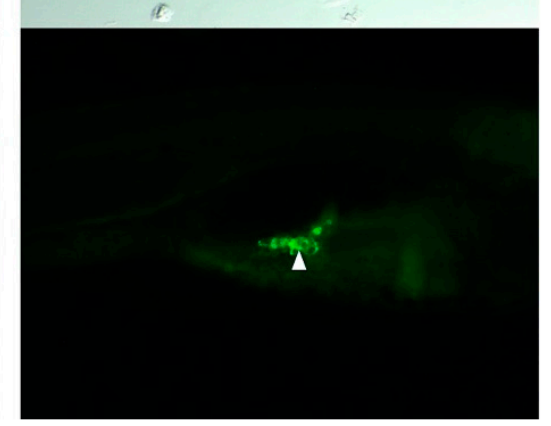

Figure 3. Cont. 

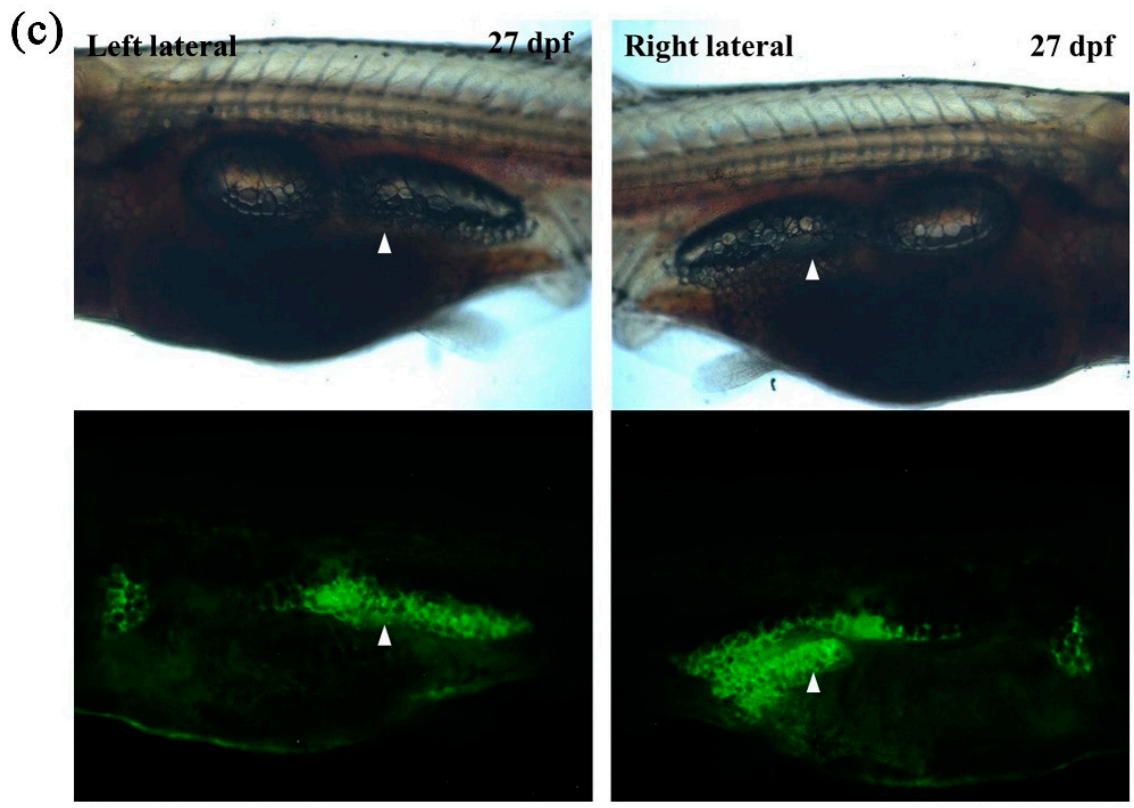

Figure 3. In vivo monitoring of visceral adipose tissue development in larvae using transgenic zebrafish. (a) Representative images of the first adipocyte emerging in three independent larvae at $10 \mathrm{dpf}$. (b) Representative images of visceral adipose tissues at $12 \mathrm{dpf}$. The first adipocyte of left lateral emerged. (c) Representative images of visceral adipose tissues at $27 \mathrm{dpf}$. White arrowheads indicate visceral adipose tissue.

\subsection{Transgenic Zebrafish Could Be Used to Monitor Nutrition-Dependent Adipocyte Development and Maintenance In Vivo}

To verify the utility of the newly generated transgenic zebrafish in live imaging, we examined the relationship between nutrition and adipocyte development. First, we tested whether food supply influenced on the appearance of the first adipocyte relative to body growth. We applied different feeding regimes and found that the group of zebrafish that received $12 \mathrm{~h}$-feeding per day developed the first adipocyte as early as $9 \mathrm{dpf}$ and at $10.7 \mathrm{dpf}$ on average (Figure $4 \mathrm{a}$ ). On the other hand, the group that was subjected to a $4 \mathrm{~h}$ - feeding regime developed the first adipocyte at $16.8 \mathrm{dpf}$ on average (Figure $4 \mathrm{a}$ ), suggesting that adipocyte development may be dependent on nutrition. Interestingly, although zebrafish belonging to different groups showed differential growth rates, the average body length of zebrafish when their first adipocytes were detected was not different $(p>0.05$, Figure $4 b)$. These results indicate that body length is the determining factor for the appearance of the first adipocyte, whereas nutrition regulates the growth rate during zebrafish development.

We further investigated the relationship between nutrition and adipocyte maintenance through live imaging of transgenic $\operatorname{Tg}$ (fabp11a: EGFP) zebrafish. We found that visceral adipose tissues gradually disappeared after withdrawal of food supply and reappeared after refeeding (Figure 4c). In particular, we found that adipose tissues were essentially undetectable after 8 days of fasting, and that they were reestablished within 2 days after refeeding (Figure 4c). Notably, starvation blocked adipocyte development in zebrafish, as we did not find any newly emerging adipocytes (Figure 4c). 
(a)

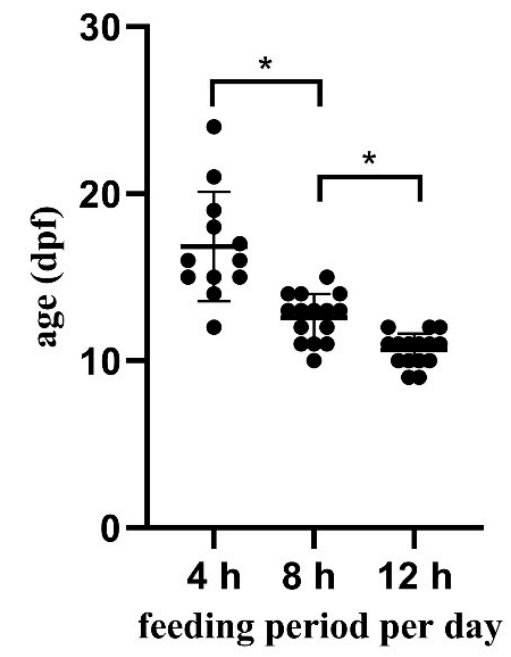

(c)
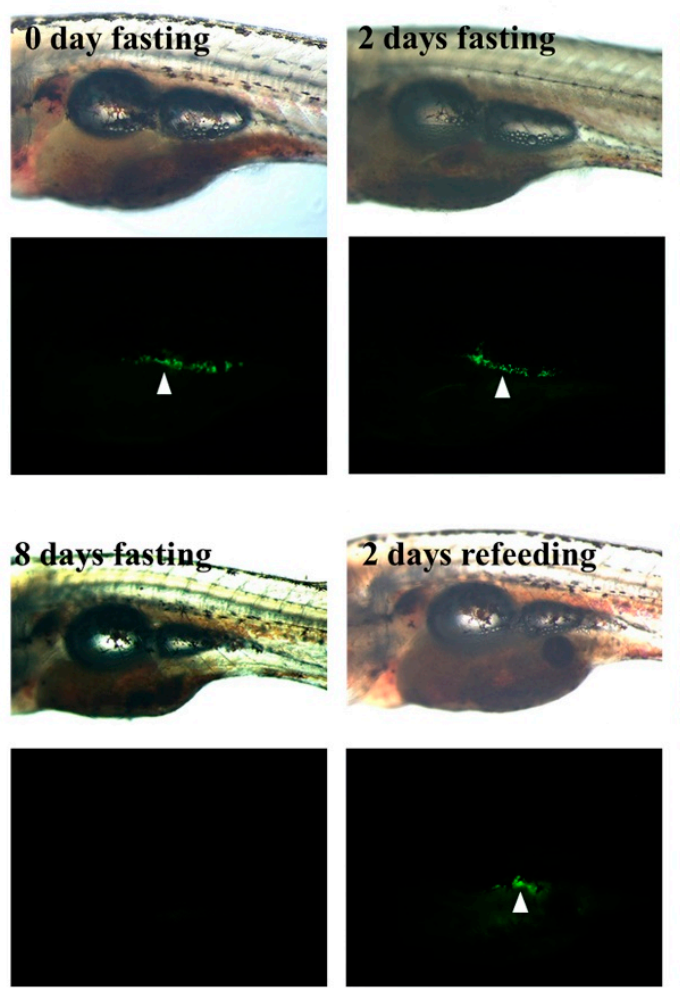

(b)
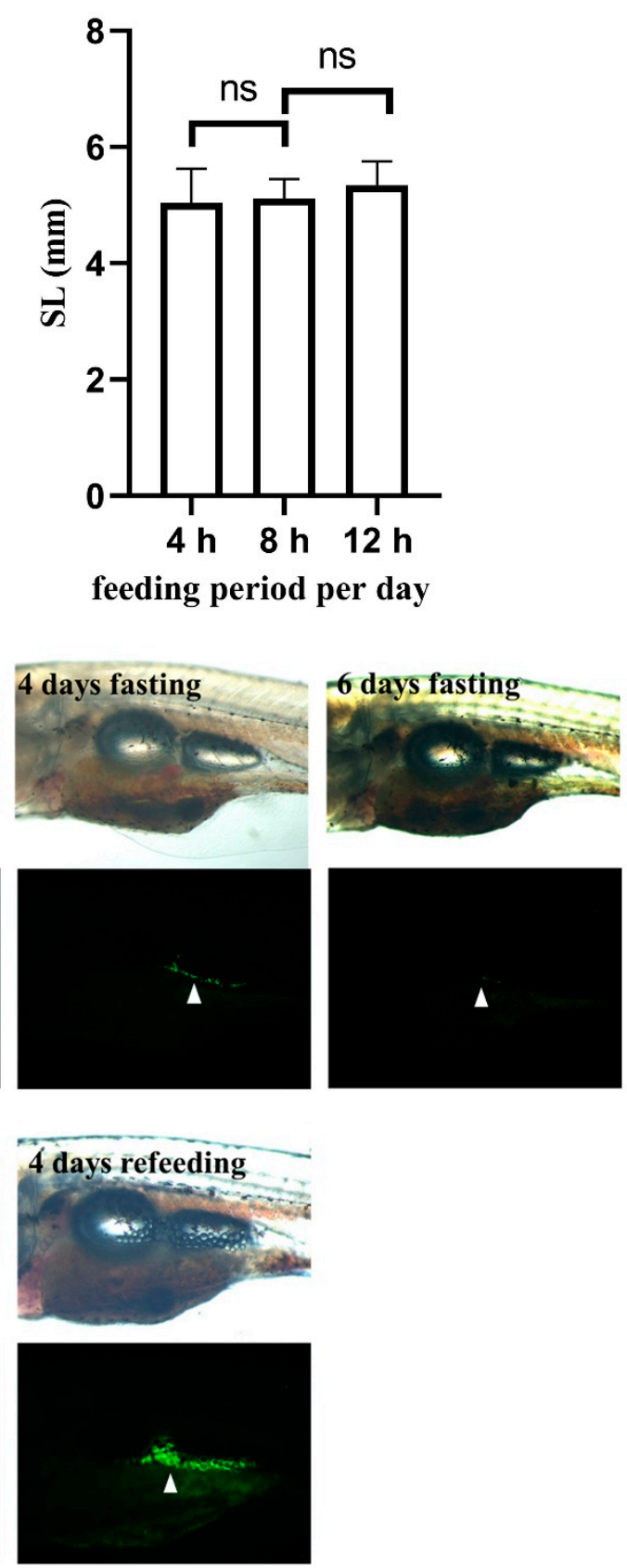

Figure 4. Nutrition influences on adipocyte development and maintenance. (a,b). Zebrafish embryos at 5 dpf were fed for different durations per day as indicated, and adipocyte development was examined. $n \geq 12$. Repeat $=3$. ${ }^{*}$ indicates $p<0.05$, and ns indicates $p>0.05$. (a) Graphical presentation of the age of larvae that developed the first adipocyte in three groups. (b) Graphical presentation of the body length of larvae that developed the first adipocyte in three groups. ns, not significant. (c) $16 \mathrm{dpf}$ larvae were selected and subjected to fasting for 8 days, and then refed for another 4 days. Representative bright field and fluorescent images are shown to orient the larvae with visceral adipose tissues marked by white arrowheads. $n \geq 5$. Repeat $=3$.

\section{Discussion}

In this study, we generated transgenic zebrafish, $\operatorname{Tg}$ (fabp11a: EGFP), to visualize adipose tissues and adipocytes in vivo. We understand that there is currently no zebrafish model that specifically labels adipocytes in vivo; therefore, the newly generated transgenic zebrafish have tremendous potential for use in adipocyte research. We found that the 
transgenic zebrafish could detect all types of adipose tissues during adipocyte development and after maturity. In addition, we were able to detect the morphology of single adipocytes of the transgenic zebrafish; hence, this transgenic zebrafish line can be potentially used to identify the origin of adipocytes during adipocyte hyperplasia and studying the size, lipid accumulation, numbers, and transformative potential of adipocytes during the life cycle of the zebrafish.

Although one needs to be careful to interpret data obtained from an animal model due to its innate difference from human, we validated that the transgenic zebrafish were well suited for monitoring the development of adipose tissues in vivo. According to our observations, the first adipocyte emerged near the pancreatic area when the zebrafish body length exceeded $5 \mathrm{~mm}$. Interestingly, we found that the visceral adipose tissues were asymmetrically developed, which necessitated imaging of both the left and right sides of the zebrafish when adipose tissues were examined. We also validated that the transgenic zebrafish were well suited for monitoring quantitative changes in adipose tissues in vivo. In particular, a fasting-refeeding experiment using the transgenic zebrafish confirmed their utility in monitoring adipocyte development and maintenance based on the nutrition status. The utility of our transgenic zebrafish could be augmented when combined with transparent transgenic zebrafish [23]; adipose tissue development according to different nutrition status could be analyzed throughout the life cycle of zebrafish to study causal relationship between obesity and the onset and progression of NAFLD. Moreover, a seemingly paradoxical "metabolically healthy obese" [4] could also be addressed by examining inter-relationship between adipose tissues and other metabolic organs, such as liver and muscle.

In summary, transgenic zebrafish $\operatorname{Tg}$ (fabp11a: EGFP) serve as a versatile and reliable model for the long-term monitoring of adipose tissue and adipocytes in vivo. Considering the capability of a library screen combined with the power of genetics that the zebrafish model can provide, our transgenic zebrafish may be useful to advance obesity-related research and preventative medicine.

\section{Materials and Methods}

\subsection{Zebrafish Husbandry and Experimental Conditions}

Zebrafish were raised and maintained under a $14 \mathrm{~h}$ light $/ 10 \mathrm{~h}$ dark cycle using standard protocols [24]. The embryos were collected, developed in egg water (5 mM $\mathrm{NaCl}, 0.17 \mathrm{mM} \mathrm{KCl}, 0.33 \mathrm{mM} \mathrm{CaCl}_{2}, 0.33 \mathrm{mM} \mathrm{MgSO}_{4}$ ) and staged according to standard protocols [25]. For the adipocyte development experiment, larvae at 5 days post fertilization (dpf) were randomly divided into three groups. The three groups were fed commercial food and fresh artemia for 4, 8 and $12 \mathrm{~h}$, respectively, and the surplus food was removed after feeding by changing the water. For the fasting and refeeding experiment, $16 \mathrm{dpf}$ larvae were subjected to fasting for 8 days and were then fed for 4 days. All the larvae were raised in a transparent $2 \mathrm{~L}$ tank under a $14 \mathrm{~h}$ light $/ 10 \mathrm{~h}$ dark cycle, with less than 15 larvae per tank. Each experiment was performed at least twice.

\subsection{Generation of DNA Constructs and Transgenic Lines}

The 1.5 kilobase-long proximal fabp 11a promoter region was amplified from total genomic DNA extracted from zebrafish embryos at $24 \mathrm{hpf}$ using a standard PCR protocol and cloned into the pCRII-Topo vector (Invitrogen, Carlsbad, CA, USA). The primer sequences used for PCR were as follows: forward primer $5^{\prime}$-AAGGATCCCCTGGCTTGCAGTTTATAGAAGCAG-3' and reverse primer $5^{\prime}$-CGACCGGTTTTCCCGAATAATGATGCTCCTCT- $3^{\prime}$. The fabp $11 a$ promoter was excised from the pCRII-Topo-fabp11a plasmid using BamHI (NEB, Ipswich, MA, USA) and sub-cloned into the BamHI site in the Mini-Tol2-EGFP plasmid. Purified mini-Tol2-fabp11a: EGFP plasmid (25 ng) and in vitro synthesized Tol2 transposase mRNA (50 ng) was microinjected into embryos at the 1-cell stage. All the injected embryos were raised and mated with wild type (WT) zebrafish to generate F1 zebrafish. F1 zebrafish 
with green fluorescence were observed and collected at various larval stages using a Leica M165FC microscope. F2 and F3 zebrafish were also generated and imaged in this study.

\subsection{Nile Red Staining and Imaging}

In vivo Nile Red staining was performed as previously described [26]. Briefly, Nile Red (Invitrogen, Carlsbad, CA, USA) was diluted to a final working concentration of $0.5 \mu \mathrm{g} / \mathrm{mL}$ egg water and then used for staining in the dark for $30 \mathrm{~min}$. At the end of staining, larvae were anesthetized with $0.03 \%$ tricaine (Sigma-Aldrich, St. Louis, MO, USA), mounted in 3\% methylcellulose, and imaged using a confocal microscope (Olympus IX81, Fluoview FV1000).

\subsection{In Vivo Imaging and Body Length Measurement}

Zebrafish larvae were anesthetized with 0.05\% tricaine (Sigma-Aldrich, St. Louis, MO, USA) in egg water and then mounted in 3\% methylcellulose for imaging. Imaging was performed using a Leica M165FC microscope, equipped with $488 \mathrm{~nm}$ laser. After imaging, the zebrafish were released into fresh water and recovered quickly. The distance between the tip of the snout and the caudal peduncle was measured and used as the larval standard length (SL) using ImageJ software (NIH, Bethesda, MD, USA).

\subsection{Statistical Analyses}

All values are reported as mean \pm standard errors of the mean (SEM). The Student's unpaired t-test in GraphPad Prism 8 software (GraphPad, San Diego, CA, USA) was used to determine statistical significance. Significance was set at $p<0.05$.

Author Contributions: Conceptualization, S.-K.C. and S.K. (SeongAe Kwak); methodology, Y.M. and K.H., S.K. (SeongJin Kim) and I.-K.N.; formal analysis, Y.M., W.L., L.L. and Y.X.; investigation, Y.M., K.H. and I.-K.N.; data curation, Y.M., K.H., W.L., L.L. and Y.X.; writing-original draft preparation, Y.M. and S.K. (SeongAe Kwak); writing—review and editing, S.-K.C. and S.K. (SeongAe Kwak); supervision, S.-K.C. and S.K.; funding acquisition, S.-K.C. and S.K. (SeongAe Kwak). All authors have read and agreed to the published version of the manuscript.

Funding: This study was supported by grants, NRF-2018R1D1A1B07050552 to S.K. (SeongAe Kwak) and NRF-2019R111A3A01061491 to S.-K.C.

Institutional Review Board Statement: The zebrafish experiment was approved on 3 December 2015 by the Committee for Ethics in Animal Experiments of Wonkwang University (WKU15-151) and carried out according to the guidelines for Animal Experiments.

Informed Consent Statement: Not applicable.

Data Availability Statement: Not applicable.

Acknowledgments: We thank members of S.-K.C. laboratory for sharing materials and providing constructive comments on this project.

Conflicts of Interest: The authors declare no conflict of interest.

\section{References}

1. Blüher, M. Obesity: Global epidemiology and pathogenesis. Nat. Rev. Endocrinol. 2019, 15, 288-298. [CrossRef] [PubMed]

2. Mandviwala, T.; Khalid, U.; Deswal, A. Obesity and Cardiovascular Disease: A Risk Factor or a Risk Marker? Curr. Atheroscler. Rep. 2016, 18, 21. [CrossRef] [PubMed]

3. Liu, T.; Sun, Y.-C.; Cheng, P.; Shao, H.-G. Adipose tissue macrophage-derived exosomal miR-29a regulates obesity-associated insulin resistance. Biochem. Biophys. Res. Commun. 2019, 515, 352-358. [CrossRef]

4. Tarantino, G.; Citro, V.; Capone, D. Nonalcoholic Fatty Liver Disease: A Challenge from Mechanisms to Therapy. J. Clin. Med. 2019, 9, 15. [CrossRef] [PubMed]

5. Johnson, A.M.F.; Olefsky, J.M. The origins and drivers of insulin resistance. Cell 2013, 152, 673-684. [CrossRef]

6. Furukawa, S.; Fujita, T.; Shimabukuro, M.; Iwaki, M.; Yamada, Y.; Nakajima, Y.; Nakayama, O.; Makishima, M.; Matsuda, M.; Shimomura, I. Increased oxidative stress in obesity and its impact on metabolic syndrome. J. Clin. Investig. 2004, 114, 1752-1761. [CrossRef] [PubMed] 
7. Satapati, S.; Kucejova, B.; Duarte, J.A.G.; Fletcher, J.A.; Reynolds, L.; Sunny, N.E.; He, T.; Nair, L.A.; Livingston, K.A.; Livingston, K.; et al. Mitochondrial metabolism mediates oxidative stress and inflammation in fatty liver. J. Clin. Investig. 2015, 125, 4447-4462. [CrossRef]

8. Muñoz-Garach, A.; Cornejo-Pareja, I.; Tinahones, F.J. Does Metabolically Healthy Obesity Exist? Nutrients 2016, 8, 320. [CrossRef] [PubMed]

9. Virtue, S.; Vidal-Puig, A. Adipose tissue expandability, lipotoxicity and the Metabolic Syndrome-an allostatic perspective. Biochim. Biophys. Acta 2010, 1801, 338-349. [CrossRef]

10. Hirosumi, J.; Tuncman, G.; Chang, L.; Görgün, C.Z.; Uysal, K.T.; Maeda, K.; Karin, M.; Hotamisligil, G.S. A central role for JNK in obesity and insulin resistance. Nature 2002, 420,333-336. [CrossRef]

11. Elemans, L.M.H.; Cervera, I.P.; Riley, S.E.; Wafer, R.; Fong, R.; Tandon, P.; Minchin, J.E.N. Quantitative analyses of adiposity dynamics in zebrafish. Adipocyte 2019, 8, 330-338. [CrossRef] [PubMed]

12. Gut, P.; Reischauer, S.; Stainier, D.Y.R.; Arnaout, R. Little fish, big data: Zebrafish as a model for cardiovascular and metabolic disease. Physiol. Rev. 2017, 97, 889-938. [CrossRef]

13. Imrie, D.; Sadler, K.C. White adipose tissue development in zebrafish is regulated by both developmental time and fish size. Dev. Dyn. 2010, 239, 3013-3023. [CrossRef] [PubMed]

14. Song, Y.; Cone, R.D. Creation of a genetic model of obesity in a teleost. FASEB J. 2007, 21, 2042-2049. [CrossRef] [PubMed]

15. Flynn, E.J.; Trent, C.M.; Rawls, J.F. Ontogeny and nutritional control of adipogenesis in zebrafish (Danio rerio). J. Lipid Res. 2009, 50, 1641-1652. [CrossRef] [PubMed]

16. Minchin, J.E.N.; Rawls, J.F. In vivo analysis of white adipose tissue in zebrafish. Methods Cell Biol. 2011, 105, 63-86. [CrossRef] [PubMed]

17. Oku, H.; Umino, T. Molecular characterization of peroxisome proliferator-activated receptors (PPARs) and their gene expression in the differentiating adipocytes of red sea bream Pagrus major. Comp. Biochem. Physiol. B Biochem. Mol. Biol. 2008, 151, 268-277. [CrossRef]

18. Michel, M.; Page-McCaw, P.S.; Chen, W.; Cone, R.D. Leptin signaling regulates glucose homeostasis, but not adipostasis, in the zebrafish. Proc. Natl. Acad. Sci. USA 2016, 113, 3084-3089. [CrossRef]

19. Minchin, J.E.N.; Rawls, J.F. A classification system for zebrafish adipose tissues. Dis. Model. Mech. 2017, 10, 797-809. [CrossRef]

20. Den Broeder, M.J.; Kopylova, V.A.; Kamminga, L.M.; Legler, J. Zebrafish as a Model to Study the Role of Peroxisome ProliferatingActivated Receptors in Adipogenesis and Obesity. PPAR Res. 2015, 2015, 358029. [CrossRef]

21. Karanth, S.; Denovan-Wright, E.M.; Thisse, C.; Thisse, B.; Wright, J.M. The evolutionary relationship between the duplicated copies of the zebrafish fabp11 gene and the tetrapod FABP4, FABP5, FABP8 and FABP9 genes. FEBS J. 2008, 275, 3031-3040. [CrossRef]

22. Liu, R.-Z.; Saxena, V.; Sharma, M.K.; Thisse, C.; Thisse, B.; Denovan-Wright, E.M.; Wright, J.M. The fabp4 gene of zebrafish (Danio rerio)-Genomic homology with the mammalian FABP4 and divergence from the zebrafish fabp3 in developmental expression. FEBS J. 2007, 274, 1621-1633. [CrossRef] [PubMed]

23. White, R.M.; Sessa, A.; Burke, C.; Bowman, T.; LeBlanc, J.; Ceol, C.; Bourque, C.; Dovey, M.; Goessling, W.; Burns, C.E.; et al. Transparent adult zebrafish as a tool for in vivo transplantation analysis. Cell Stem Cell 2008, 2, 183-189. [CrossRef] [PubMed]

24. Westerfield, M. The Zebrafish Book, a Guide for the Laboratory Use of Zebrafish (Danio Rerio), 5th ed.; University of Oregon Press: Eugene, OP, USA, 2007.

25. Kimmel, C.B.; Ballard, W.W.; Kimmel, S.R.; Ullmann, B.; Schilling, T.F. Stages of embryonic development of the zebrafish. Dev. Dyn. 1995, 203, 253-310. [CrossRef] [PubMed]

26. Minchin, J.E.N.; Rawls, J.F. In vivo imaging and quantification of regional adiposity in zebrafish. Methods Cell Biol. 2017, 138, 3-27. [CrossRef] 